\title{
A Relativistic Coupled-Channel Formalism for the Pion Form Factor
}

\author{
E.P. Biernat ${ }^{1, a}$, W. Schweiger ${ }^{1}$, K. Fuchsberger ${ }^{2}$, and W.H. Klink ${ }^{3}$ \\ 1 Institut für Physik, Universität Graz, A-8010 Graz, Austria \\ 2 BE-OP Division, CERN, CH-1211 Geneve 23, Switzerland \\ 3 Department of Physics and Astronomy, University of Iowa, Iowa City, Iowa, USA
}

\begin{abstract}
The electromagnetic form factor of a confined quark-antiquark pair is calculated within the framework of point-form relativistic quantum mechanics. The dynamics of the exchanged photon is explicitly taken into account by treating the electromagnetic scattering of an electron by a meson as a relativistic two-channel problem for a Bakamjian-Thomas type mass operator. This approach guarantees Poincaré invariance. Using a Feshbach reduction the coupled-channel problem can be converted into a one-channel problem for the elastic electron-meson channel. By comparing the one-photon-exchange optical potential at the constituent and hadronic levels, we are able to unambiguously identify the electromagnetic meson form factor. Violations of cluster-separability properties, which are inherent in the Bakamjian-Thomas approach, become negligible for sufficiently large invariant mass of the electron-meson system. In the limit of an infinitely large invariant mass, an equivalence with form-factor calculations done in front-form relativistic quantum mechanics is established analytically.
\end{abstract}

\section{Introduction}

The invariant electron-hadron scattering amplitude in the one-photon-exchange approximation can be written as the contraction of a (point-like) electron current with a hadron current times the photon propagator. The hadron current is a sum of independent Lorentz covariants, which transform like four-vectors and are multiplied by Lorentz invariant functions, the hadron form factors. These form factors, which are functions of the four-momentum-transfer squared $\left(q^{2}=-Q^{2}\right)$, are the observables that describe the electromagnetic structure of the hadron. The present calculation of meson form factors is based on the point form of relativistic quantum mechanics. This form is characterized by the property that all four generators of space-time translations are interaction dependent, whereas the generators of Lorentz transformations stay free of interactions.

Our strategy is to start with a Poincaré invariant treatment of electron-meson scattering. To this aim we adopt a two-channel version of the Bakamjian-Thomas (BT) approach $[1,2]$ and work within a velocity-state representation [3], which is particularly convenient for our purposes. The second channel contains the photon. Its coupling to electrons, hadrons and quarks can be taken from conventional quantum electrodynamics [4], but has to be appropriately adapted to be applicable within the BT-framework. In the following we will outline how this formalism works at the hadronic level for the scattering of an electron by a (spatially extended) pseudoscalar meson. Then we will proceed in an analogous way at the constituent level. Observing that the one-photon-exchange optical potential at

a e-mail: elmar.biernat@uni-graz.at both the hadronic and the constituent levels have the structure of a current-current interaction then makes it possible to extract the meson current and, consequently, the meson form factor.

\section{One-Photon-Exchange Optical Potential}

If the dynamics of the exchanged photon is to be taken into account explicitly, the mass operator for electromagnetic scattering of an electron by a pseudoscalar meson should act on a direct sum of Hilbert spaces that accommodate the initial and final electron-meson states as well as the intermediate electron-meson-photon states. By projecting the mass eigenstates onto these subspaces, the eigenvalue equation for the mass operator becomes a system of two coupled equations for the respective components. Elimination of the component containing the photon then leads to a single equation for the electron-meson component $\left|\psi_{e M}\right\rangle$ :

$$
\begin{aligned}
\left(\hat{M}_{e M}-m\right)\left|\psi_{e M}\right\rangle & =\hat{K}^{\dagger}\left(\hat{M}_{e M \gamma}-m\right)^{-1} \hat{K}\left|\psi_{e M}\right\rangle \\
& =: \hat{V}_{\mathrm{opt}}(m)\left|\psi_{e M}\right\rangle .
\end{aligned}
$$

Here $m$ is the mass eigenvalue, $\hat{K}$ and $\hat{K}^{\dagger}$ are vertex operators responsible for the emission and absorption of a photon and $\hat{M}_{e M}$ and $\hat{M}_{e M \gamma}$ are mass operators for the free electron-meson and electron-meson-photon systems, respectively. The right-hand side of Eq. (1) describes the action of the one-photon-exchange optical potential $\hat{V}_{\text {opt }}$ on the $\left|\psi_{e M}\right\rangle$ state. To extract the meson current we calculate matrix elements of the optical potential $\hat{V}_{\text {opt }}$ in a velocity state basis [3]. On the hadronic level the structure of 
the meson is encoded in a phenomenological form factor $f(Q)$ which is inserted by hand at the photon-meson vertex. Furthermore, in order to be able to work within the BT framework [1] one has to resort to the approximation that the four-velocity of the whole $(e M-e M \gamma)$ system is conserved at the electromagnetic vertices. With these assumptions and neglecting self-energy contributions due to photons being emitted and absorbed by the same particle the on-shell matrix elements of the optical potential on the hadronic level read [5]

$$
\begin{aligned}
& \left\langle v^{\prime} ; \mathbf{k}_{e}^{\prime}, \mu_{e}^{\prime} ; \mathbf{k}_{M}^{\prime}\left|\hat{V}_{\mathrm{opt}}(m)\right| v ; \mathbf{k}_{e}, \mu_{e} ; \mathbf{k}_{M}\right\rangle_{\mathrm{on}-\text { shell }} \\
& \propto v_{0} \delta^{3}\left(\mathbf{v}^{\prime}-\mathbf{v}\right) f(Q) j_{\mu}\left(\mathbf{k}_{M}^{\prime} ; \mathbf{k}_{M}\right) \frac{\left(-\mathrm{g}^{\mu \nu}\right)}{Q^{2}} j_{v}\left(\mathbf{k}_{e}^{\prime}, \mu_{e}^{\prime} ; \mathbf{k}_{e}, \mu_{e}\right) .
\end{aligned}
$$

Here $v^{(\prime)}$ is the incoming (outgoing) total velocity of the electron-meson system, $\mathbf{k}_{e}^{(\prime)}$ and $\mathbf{k}_{M}^{(\prime)}=-\mathbf{k}_{e}^{(\prime)}$ are the incoming (outgoing) center-of-mass momenta of the electron and meson, respectively. "On-shell" means that due to the center-of-mass kinematics and the fact that we are only interested in the one-photon-exchange amplitude we can set $m=\left(m_{e}^{2}+\mathbf{k}_{e}^{2}\right)^{1 / 2}+\left(m_{M}^{2}+\mathbf{k}_{M}^{2}\right)^{1 / 2}$ and $\left|\mathbf{k}_{M}\right|=\left|\mathbf{k}_{M}^{(\prime)}\right|=\left|\mathbf{k}_{e}\right|=$ $\left|\mathbf{k}_{e}^{(\prime)}\right|$. The $\mu_{e}$ 's are the spin-projections of the electron and $j_{v}\left(\mathbf{k}_{e}^{\prime}, \mu_{e}^{\prime} ; \mathbf{k}_{e}, \mu_{e}\right)$ as well as $j_{\mu}\left(\mathbf{k}_{M}^{\prime} ; \mathbf{k}_{M}\right)$ are the point-like electromagnetic electron and meson currents, respectively. Apart from a kinematical factor (which has been dropped for better readability) the right-hand side of Eq. (2) corresponds to the familiar one-photon exchange amplitude for elastic electron-meson scattering (calculated in the centerof-mass system).

In order to achieve a microscopic description of the form factor $f(Q)$ we employ a constituent-quark model in which the meson is composed of a quark and an antiquark. Working within the same approach as outlined above, the mass operator has now to be defined on a Hilbert space that comprises electron-quark-antiquark and electron-quark-antiquark-photon states. Furthermore we assume that an instantaneous confinement potential acts between quark and antiquark (in both channels) and, as before, the total velocity should be conserved at the photon-electron and photon(anti)quark vertices. For a comparison with Eq. (2) one has to calculate (on-shell) matrix elements of the optical potential on the constituent-level, $\hat{V}_{\mathrm{opt}}^{\text {const }}$, between quarkantiquark bound states with the quantum numbers of the meson. Neglecting again self-energy contributions a tedious calculation yields [5]:

$$
\begin{aligned}
& \left\langle v^{\prime} ; \mathbf{k}_{e}^{\prime}, \mu_{e}^{\prime} ; \mathbf{k}_{M}^{\prime}\left|\hat{V}_{\mathrm{opt}}^{\mathrm{const}}(m)\right| v ; \mathbf{k}_{e}, \mu_{e} ; \mathbf{k}_{M}\right\rangle_{\mathrm{on}-\text { shell }} \\
& \propto v_{0} \delta^{3}\left(\mathbf{v}^{\prime}-\mathbf{v}\right) J_{\mu}\left(\mathbf{k}_{M}^{\prime} ; \mathbf{k}_{M}\right) \frac{\left(-\mathrm{g}^{\mu v}\right)}{Q^{2}} j_{v}\left(\mathbf{k}_{e}^{\prime}, \mu_{e}^{\prime} ; \mathbf{k}_{e}, \mu_{e}\right) .
\end{aligned}
$$

Here we have dropped the same kinematical factor as in Eq. (2). The microscopic meson current $J_{v}\left(\mathbf{k}_{M}^{\prime} ; \mathbf{k}_{M}\right)$ is a rather lengthy expression of an integral over bound state wave functions, quark currents and Wigner $D$ functions [6].

\section{Meson Form Factor}

If the quark-antiquark bound state at the hadronic level has the same quantum numbers as the meson, we can equate the right-hand sides of Eqs. (2) and (3) to find an expression for the form factor [5]

$$
f\left(Q,\left|\mathbf{k}_{M}\right|\right)=\frac{j^{\mu}\left(\mathbf{k}_{e}^{\prime}, \mu_{e}^{\prime} ; \mathbf{k}_{e}, \mu_{e}\right) J_{\mu}\left(\mathbf{k}_{M}^{\prime} ; \mathbf{k}_{M}\right)}{j^{v}\left(\mathbf{k}_{e}^{\prime}, \mu_{e}^{\prime} ; \mathbf{k}_{e}, \mu_{e}\right) j_{v}\left(\mathbf{k}_{M}^{\prime} ; \mathbf{k}_{M}\right)} .
$$

Here we have introduced the magnitude of the center-ofmass momentum of the meson $\left|\mathbf{k}_{M}\right|$ as a further argument of the vertex form factor. This additional dependence of the form factor on $\left|\mathbf{k}_{M}\right|$ (or equivalently the invariant mass of the electron-meson system) is a consequence of velocity conservation at the electromagnetic vertices, which is required to get a BT-type mass operator. It should be emphasized that such an additional $\left|\mathbf{k}_{M}\right|$-dependence of the meson form factor still preserves Poincaré invariance. But it violates cluster separability. Violation of cluster separability, in particular the violation of the cluster condition for the Poincaré generators, is nearly unavoidable within the BT-framework, but can be overcome by the introduction of, so called, "packing operators" [2]. ${ }^{1}$ Fortunately this $\left|\mathbf{k}_{M}\right|$-dependence vanishes rather quickly with increasing $\left|\mathbf{k}_{M}\right|$ and suggests taking the limit $\left|\mathbf{k}_{M}\right| \rightarrow \infty$. In this limit the microscopic meson current factorizes explicitly into a point-like meson current times a form factor:

$$
J_{v}\left(\mathbf{k}_{M}^{\prime} ; \mathbf{k}_{M}\right) \stackrel{\left|\mathbf{k}_{M}\right| \rightarrow \infty}{\longrightarrow} F(Q) j_{v}\left(\mathbf{k}_{M}^{\prime} ; \mathbf{k}_{M}\right) .
$$

The final result for the form factor has a simple analytical form [6]:

$$
F(Q)=\int \mathrm{d}^{3} \tilde{k}_{q}^{\prime} \sqrt{\frac{m_{q \bar{q}}}{m_{q \bar{q}}^{\prime}}} \mathcal{S} \Psi^{*}\left(\tilde{\mathbf{k}}_{q}^{\prime}\right) \Psi\left(\tilde{\mathbf{k}}_{q}\right) .
$$

Here $Q^{2}=\mathbf{q}^{2}$ is the momentum transfer squared with $\mathbf{q}=\mathbf{k}_{q}^{\prime}-\mathbf{k}_{q}=\mathbf{k}_{M}^{\prime}-\mathbf{k}_{M}$ and $m_{q \bar{q}}^{2}=\left(E_{q}+E_{\bar{q}}\right)^{2}-\mathbf{k}_{M}^{2}$ is the invariant mass of the quark-antiquark pair. Quantities without a tilde refer to the electron-meson center-of-mass and quantities with a tilde to the meson rest system. $\Psi$ is the bound-state wave function of the quark-antiquark pair and $\mathcal{S}$ is a spin-rotation factor which takes into account the substantial effect of the quark spin on the form factor. By an appropriate change of variables the integral for the form factor, Eq. (6), takes the same form as the integral for the pion form factor from front-form calculations $[9,10]$. This remarkable result means that relativity is treated in an equivalent way and the physical ingredients are the same in both approaches.

For a simple two-parameter harmonic-oscillator wave function with the parametrization taken from $[9,10]$ our result for the pion electromagnetic form factor provide a reasonable fit to the data as shown in Fig. 1.

\footnotetext{
${ }^{1}$ It should be noted that for the case of an interacting pair and a spectator our velocity-state representation of operators does not violate cluster separability for the scattering operator [7]. The separability condition for the scattering operator should, however, be contrasted with the stronger condition for the generators [8].
} 


\section{$19^{\text {th }}$ International IUPAP Conference on Few-Body Problems in Physics}

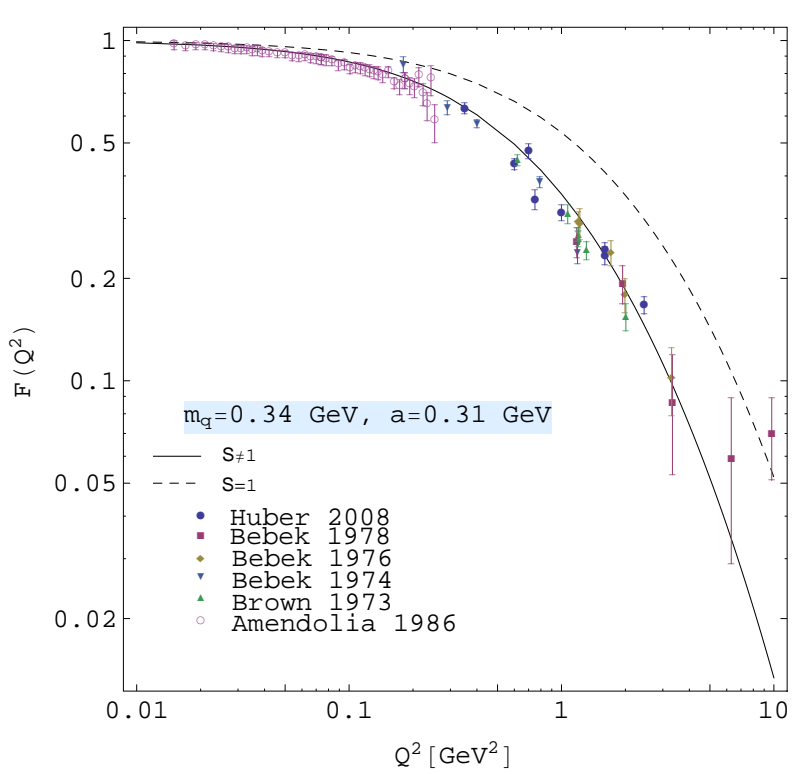

Fig. 1. $Q^{2}$-dependence of the pion form factor with (solid) and without (dashed) spin-rotation factor $\mathcal{S}$. Values for the quark mass $m_{q}$ and the oscillator parameter $a$ are taken from $[9,10]$ and data are taken from [11-16].

\section{Summary and Outlook}

We have applied the point form of relativistic quantum mechanics in connection with the BT-formalism to analyze the electromagnetic structure of pseudoscalar mesons. In our approach the scattering of an electron by a confined quark-antiquark pair is treated as a two-channel problem for the mass operator. In this way the dynamics of the exchanged photon can be taken into account explicitly. Quark confinement is treated via an instantaneous potential. The emission and absorption of a photon by an electron or (anti-)quark is described by a vertex interaction that has the Lorentz structure of the field theoretical vertex but conserves the four-velocity of the whole system. By construction this approach is Poincaré invariant. We are then able to identify the meson form factor from the one-photon-exchange optical potential in an unambiguous way. The extracted meson form factor, however, depends not only on Mandelstam $t=-Q^{2}$ (the four-momentum transfer squared) but also shows a (mild) dependence on Mandelstam $s$ (the total invariant mass squared of the $e M$ system). This additional $s$-dependence does not spoil Poincaré invariance but indicates a violation of cluster separability. It is a consequence of working with the point-form version of the BT-formalism which demands velocity conservation at each interaction vertex. The observation that the $s$-dependence vanishes quickly with increasing $s$ indicates, however, that cluster-separability-violating effects are negligible for sufficiently large $s .{ }^{2}$ Indeed, as we show

\footnotetext{
${ }^{2}$ In point form it seems natural to use Lorentz boosts in opposite directions to space-like separate subsystems [17]. In this way one stays on the quantization hypersurface. Separations by boosts, however, increases the invariant mass of the whole sys-
}

analytically, in the limit of infinitely large $s$ the microscopic meson current goes over into a product of the usual point-like meson current times an integral with the integrand depending only on the momentum transfer $Q$ and on internal variables (that are integrated and summed over). In this limit the form factor acquires a simple analytical form which has been shown to be equivalent to the usual front-form expression in a $q^{+}=0$ frame.

Our multichannel approach for the calculation of form factors can easily be generalized to electroweak form factors of arbitrary few-body bound systems. By an appropriate extension of the Hilbert space it should also be possible to handle exchange-current effects within this type of approach.

\section{References}

1. B. Bakamjian and L.H. Thomas, Phys. Rev. 92 (1953) 1300

2. B.D. Keister and W.N. Polyzou, Adv. Nucl. Phys. 20 (1991) 225

3. W.H. Klink, Phys. Rev. C58 (1998) 3617

4. W.H. Klink, Nucl. Phys. A716 (2003) 123

5. K. Fuchsberger, Master's thesis, Karl-FranzensUniversität Graz (2007)

6. E.P. Biernat, W. Schweiger, K. Fuchsberger, and W.H. Klink, Phys. Rev. C79 (2009) 055203

7. F. Coester, Helv. Phys. Acta 38 (1965) 7

8. F. Coester, and W.N. Polyzou, Phys. Rev. D26 (1982) 1348

9. P.L. Chung, F. Coester, and W.N. Polyzou, Phys. Lett. B205 (1988) 545

10. F. Coester, W.N. Polyzou, Phys. Rev. C71 (2005) 028202

11. S.R. Amendolia et al., Nucl. Phys. B277 (1986) 168

12. C.N. Brown et al., Phys. Rev. D8 (1973) 92

13. C.J. Bebek et al., Phys. Rev. D9 (1974) 1229

14. C.J. Bebek et al., Phys. Rev. D13 (1976) 25

15. C.J. Bebek et al., Phys. Rev. D17 (1978) 1693

16. G.M. Huber et al., Phys. Rev. C78 (2008) 045203

17. E.P. Biernat, W.H. Klink and W. Schweiger, paper in preparation tem. Or, reversing the argument, by taking $s$ large we separate the electron from the meson such that it does not affect the meson structure. 